\title{
Fragebogen
}

1 Welche Aussage ist richtig? Atopische Kleinkinder sollten

A aufgrund möglicher anaphylaktischer Reaktionen nicht geimpft werden

B bis zum Schulalter angesichts nicht konsistenter Ergebnisse keine Allergietests machen

C möglichst frühzeitig und regelmäßig Tierhaarallergenen ausgesetzt werden, um das Immunsystem zu trainieren

D bei rezidivierenden obstruktiven Bronchitiden generell keiner medikamentösen Therapie unterzogen werden, um den Organismus nicht unnötig zu belasten.

E als nicht beziehungsweise nur partiell gestillte Säuglinge mit Hydrolysatnahrungen ernährt werden

Welche Aussagen sind richtig? Die subkutane spezifische Immuntherapie (SCIT)

1 kann bei suffizienter allergologischer Diagnostik bereits bei Kindern durchgeführt werden

2 ist als wirksame Maßnahme sekundärer Allergieprävention definiert

3 ist für die allergische Rhinokonjunktivitis bei Pollen- und Hausstaubmilbenallergie durch zahlreiche kontrollierte Studien evidenzbasiert

4 verhindert das Risiko von Neusensibilisierungen und Etagenwechsel bei Pollenallergikern

5 bewirkt eine Abnahme klinischer Symptome und nötiger Medikation

6 ist bei allergischem Asthma als Therapieoption neben Allergenkarenz und Pharmakotherapie empfehlenswert

A Nur Aussagen 1, 2 und 4 sind richtig

B Nur Aussagen 2, 3 und 5 sind richtig

C Nur Aussagen 3, 5 und 6 sind richtig

D Nur Aussagen 1, 4 und 6 sind richtig

E Alle Aussagen sind richtig

3 Welche Aussage trifft zu?

A Bauernkinder leiden häufiger an Allergien als Stadtkinder

B Allergische Erkrankungen haben einen geringen sozio-ökonomischen Stellenwert

C die wichtigsten allergischen Erkrankungen sind Heuschnupfen und Nahrungsmittelallergien

D Ambrosia ist nur in Osteuropa als Allergieauslöser bedeutsam

E die „Hygienehypothese“ ist eine mögliche Erklärung für die Zunahme allergischer Erkrankungen
4 Welche Aussagen sind falsch?

1 Encasings werden bei Hausstaubmilbensensibilisierung nicht mehr empfohlen

2 in der Therapie des Asthma bronchiale sollen inhalative Steroide frühzeitig eingesetzt werden

3 der Anti-IgE-Antikörper Omalizumab ist für Patienten mit schwerem allergischem Asthma bronchiale als Zusatztherapie zugelassen

4 die sublinguale Immuntherapie hat den gleichen Stellenwert wie die subkutane Immuntherapie

5 die spezifische Immuntherapie sollte bei mangelnder Patientencompliance nicht durchgeführt werden

A Antworten 1 und 4 sind falsch

B Antworten 2, 3 und 5 sind falsch

C Antworten 2 und 4 sind falsch

D Antworten 2 und 5 sind falsch

E Antworten 3 und 4 sind falsch

5 Welche Aussage zur Pseudoallergie ist falsch?

A zu den Symptomen gehören Kopfund Bauchschmerzen, Schwindel, Übelkeit und Bronchokonstriktion

B Symptome können bereits beim ersten Kontakt auftreten

C kann durch Allergietestung gesichert werden

D ist dosisabhängig

E zu den möglichen Auslösern zählen Fisch, Wurst, Käse und Alkohol

6 Welche Aussage trifft zu?

A Antihistaminika sind wirksam bei allen Formen der Pseudoallergie

B die Diaminooxidase ist verantwortlich für den intrazellulären Histaminabbau

C die Bestimmung der Serum Tryptase ist ohne Bedeutung für die Diagnose einer Histamin-Intoleranz

D bei einer DAO-Serumkonzentration von $>10 \mathrm{U} / \mathrm{ml}$ liegt sehr wahrscheinlich eine Histamin-Intoleranz vor

E eine Histamin-Intoleranz kann bedingt sein durch einen Mangel an Vitamin $\mathrm{B}_{6}$ und Vitamin $\mathrm{C}$

7 Welche Aussagen sind richtig?

1 Diaminooxidase kann oral substituiert werden

2 die Bestimmung der DAO-Aktivität zählt zur Routinediagnostik bei Verdacht auf Histamin-Intoleranz

3 Medikamente können eine Blockade der DAO-Aktivität bewirken

4 Histamin entsteht bei Gärungs- und Reifungsprozessen

5 Tyramin und Serotonin gehören nicht zu den biogenen Aminen
A Aussagen 1,2 und 3 sind richtig

B Aussagen 1, 2, 3 und 4 sind richtig

C Aussagen 2, 3 und 5 sind richtig

D Aussagen 1, 3 und 4 sind richtig

E Alle Aussagen sind richtig

8 Welche Aussage ist falsch?

A bereits kleine Feinstaubbedingte Veränderungen der Vitalkapazität erhöhen die Zahl der Menschen mit pathologischer Lungenfunktion erheblich

B die Entwicklung der Lunge von Kindern wird mit zunehmenden PM2.5Konzentrationen in der Luft gehemmt

C $90 \%$ des Feinstaubes der Luft stammt aus Verbrennungsmotoren

D Menschen mit asymptomatischer bronchialer Hyperreaktivität beziehungsweise mit manifestem Asthma haben das größte Risiko, Feinstaubbedingt eine COPD zu entwickeln

E moderne Dieselfilter reduzieren die PM10-Produktion von Motoren in ganz erheblichem Maße

9 Welche Aussage ist richtig?

A Zigarettenrauch stellt nur für Lungenkranke ein Problem dar

B für Feinstaubkonzentrationen existieren leider noch keine Grenzwerte

C moderne Dieselfilter haben sich leider als nur wenig wirksam erwiesen

D aufgrund statistischer Modelle muss angenommen werden, dass erhöhte Feinstaub-Konzentrationen für bis zu 500000 Asthma-Anfälle in Europa pro Jahr verantwortlich sind

E Passivrauchen erhöht die Morbidität, nicht aber die Mortalität

10 Welche Aussage ist richtig?

A Trotz Verbesserung der Motoren von Kraftfahrzeugen zeigt diese Feinstaubquelle ein stetiges Wachstum

B Im Vergleich zur Luftverschmutzung der Industrie sind die Feinstaubkonzentrationen, die beim Passivrauchen eingeatmet werden, vernachlässigbar

C Feinstaub verteilt sich sehr gleichmäBig in der Luft, sodass es in einer Stadt keine Rolle spielt, wo man wohnt

D eine gesunde Lunge kann aufgrund der mukoziliären Clearance die durchschnittlichen Feinstaubkonzentrationen in Mitteleuropa problemlos verkraften

E die hohen Feinstaubkonzentrationen stellen eine Hauptursache für die zunehmende Morbidität an COPD in Europa dar 


\begin{tabular}{|c|c|c|c|c|c|c|c|c|c|c|c|c|}
\hline \multirow{5}{*}{$\begin{array}{l}\text { Lernerfolgskontrolle } \\
\text { (nur eine Antwort pro Frage ankreuzen) }\end{array}$} & 1 & A & B & C & D & E & 6 & A & B & C & D & $E$ \\
\hline & 2 & A & B & C & D & E & 7 & A & B & C & D & $E$ \\
\hline & 3 & A & B & C & D & E & 8 & A & B & C & D & E \\
\hline & 4 & A & B & C & D & E & 9 & A & B & C & D & $\mathbf{E}$ \\
\hline & 5 & A & B & C & D & E & 10 & A & B & C & D & E \\
\hline
\end{tabular}

Ihre Adresse

Name Vorname

Straße

PLZ, Ort

Erklärung

Ich versichere, dass ich die Beantwortung der Fragen selbst und ohne fremde Hilfe durchgeführt habe.

CME-Wertmarken

für Nicht-Abonnenten
CME-Wertmarken (für Teilnehmer, die die Notfall \& Hausarztmedizin nicht abonniert haben) können beim Verlag zu folgenden Bedingungen erworben werden: 6er-Pack Thieme-CME-Wertmarken, Preis: 63,- Euro inkl. MwSt., Artikel-Nr. 901916; 12er-Pack Thieme-CME-Wertmarken, Preis: 99,- Euro inkl. MwSt., Artikel-Nr. 901917. Bitte richten Sie Bestellungen an: Georg Thieme Verlag KG, Kundenservice, Postfach 301120, 70451 Stuttgart.

Feld für CME-Wertmarke

Bitte hier Wertmarke aufkleben,

Abonnenten bitte Abonummer eintragen

Teilnahmebedingungen der zertifizierten Fortbildung

Für diese Fortbildungseinheit erhalten Sie 3 Fortbildungspunkte im Rahmen des

Fortbildungszertifikates der Ärztekammern. Hierfür:

- müssen $70 \%$ der Fragen richtig beantwortet sein.

- muss die oben stehende Erklärung sowie die Evaluation vollständig ausgefüllt sein. Unvollständig ausgefüllte Bögen können nicht berücksichtigt werden!

- muss Ihre Abonnentennummer im entsprechenden Feld des Antwortbogens angegeben oder eine CME-Wertmarke im dafür vorgesehenen Feld aufgeklebt sein.

\begin{tabular}{|c|c|c|}
\hline nis & Sie haben & Fragen richtig beantwortet und somit \\
\hline erlag ausgefüllt) & bestanden und $3 \mathrm{~F}$ & dungspunkte erworben \\
\hline
\end{tabular}

Stuttgart, (Stempel/Unterschrift)

Wichtige Hinweise

Die CME-Beiträge der Notfall \& Hausarztmedizin wurden durch die Ärztekammer Nordrhein anerkannt. Die Notfall \& Hausarztmedizin ist zur Vergabe der Fortbildungspunkte für diese Fortbildungseinheit berechtigt. Diese Fortbildungspunkte der Ärztekammer Nordrhein werden von anderen zertifizierenden Ärztekammern anerkannt. Die Vergabe der Fortbildungspunkte ist nicht an ein Abonnement gekoppelt!

Bitte senden Sie den vollständig ausgefüllten Antwortbogen und einen an Sie selbst adressierten und ausreichend frankierten Rückumschlag an den Georg Thieme Verlag KG, CME, Joachim Ortleb, Postfach 301120, 70451 Stuttgart. Einsendeschluss ist der 28. Februar 2009 (Datum des Poststempels). Die Zertifikate werden spätestens drei Wochen nach Erhalt des Antwortbogens versandt. Von telefonischen Anfragen bitten wir abzusehen. 


\section{Didaktisch-methodische Evaluation}

1 Das Fortbildungsthema kommt in meiner ärztlichen Tätigkeit

\begin{tabular}{|l|l|l|l|l|l|l}
\hline häufig vor & selten vor & regelmäßig vor & gar nor vor
\end{tabular}

2 Bezüglich des Fortbildungsthemas gab es für mich vor dem Studium dieses Beitrages

\begin{tabular}{lll} 
eine feste Gesamtstrategie $\quad$ keine Strategie noch offene Einzelprobleme: \\
\hline
\end{tabular}

3 Bezüglich des Fortbildungsthemas

fühle ich mich nach dem Studium des Beitrages in meiner Strategie bestätigt

habe ich meine Strategie geändert:

habe ich erstmals eine einheitliche Strategie erarbeitet

habe ich keine einheitliche Strategie ableiten können

4 Wurden aus der Sicht Ihrer täglichen Praxis heraus wichtige Aspekte des Themas

nicht erwähnt? Ja / Nein - wenn ja, welche:

zu knapp abgehandelt? Ja / Nein - wenn ja, welche:

überbewertet? Ja / Nein - wenn ja, welche:

5 Verständlichkeit des Beitrags

Der Beitrag ist nur für Spezialisten verständlich Der Beitrag ist auch für Nicht-Spezialisten verständlich

6 Beantwortung der Fragen

Die Fragen lassen sich aus dem Studium des Beitrages allein beantworten

Die Fragen lassen sich nur unter Zuhilfenahme zusätzlicher Literatur beantworten

7 Die Aussagen des Beitrages benötigen eine ausführlichere Darstellung

\begin{tabular}{l|l|l} 
zusätzlicher Daten $\quad$ von Befunden bildgebender Verfahren die Darstellung ist ausreichend
\end{tabular}

8 Wieviel Zeit haben Sie für das Lesen des Beitrages und die Bearbeitung des Fragebogens benötigt?

Minuten

\section{Fragen zur Person}

Ich bin Mitglied der Ärztekammer:

Jahr meiner Approbation:

Ich befinde mich in Weiterbildung zum:

Ich habe eine abgeschlossene Weiterbildung in (bitte Fach eintragen):

Ich bin tätig als:

Assistenzarzt

Chefarzt

Sonstiges

Oberarzt

Niedergelassener Arzt

Notfall \& Hausarztmedizin 2008; 34 (2): 85-87 